\title{
UPPER ESTIMATES FOR SUPERPOSITION OPERATORS AND SOME APPLICATIONS
}

\section{J. APPELL}

The application of many principles of nonlinear analysis to the study of different types of equations requires upper estimates for the operators which are generated by given functions. Such estimates are usually obtained (by elementary means) from analogous estimates that are true for these functions. In this connection it is tacitly assumed that the estimates for the operators and those for the corresponding functions are equivalent (at least in a weak sense), and this is in fact true for many types of operators in the space $C$ of continuous functions. However, for operators between the spaces $L_{p}$ and $L_{q}$ such an equivalence does not even hold for the simplest nonlinear operator, the superposition operator (Nemytskij operator). It is the aim of this paper to describe and discuss this situation in $L_{p}$ spaces. As application, certain necessary and sufficient conditions for the differentiability and asymptotic differentiability of the superposition operator are given.

\section{The case $p=q=1$}

To begin with, let us recall the following well known theorem of M. A. Krasnosel'skiǐ ([3], see also [2], [8]): Given an open subset $\Omega \subseteq \mathbf{R}^{N}$ and a Carathéodory function $f: \Omega \times \mathbf{R} \rightarrow \mathbf{R}$, the superposition operator

$$
F x(s):=f(s, x(s))
$$

maps the space $L_{1}(\Omega)$ into itself if and only if there is a pair $(a, b)$ of positive numbers such that

$$
|f(s, u)| \leqq \alpha(s)+b|u| \quad(s \in \Omega, u \in \mathbf{R})
$$

for some function $\alpha \in L_{1}(\Omega)$ with $\|\alpha\|_{1} \leqq a$. (In this case $F$ is automatically bounded and continuous.) Denote the set of such pairs $(a, b)$ associated with $f$ by $T(f)$ and let

$$
v_{f}(\varrho):=\inf \{a+b \varrho:(a, b) \in T(f)\} \quad(\varrho>0) .
$$


Moreover, we associate with the operator $F$ a function $\mu_{F}$ which is defined by

$$
\mu_{F}(\varrho):=\sup \left\{\|F x\|_{1}:\|x\|_{1} \leqq \varrho\right\} \quad(\varrho>0)
$$

and describes the growth of $F$ on balls centered at the origin.

Theorem 1. The functions $\mu_{F}$ and $v_{f}$ are equivalent in the sense that

$$
\mu_{F}(\varrho) \leqq v_{f}(\varrho) \leqq 2 \mu_{F}(\varrho) .
$$

Proof: Given $(a, b) \in T(f)$, one has immediately for $\|x\|_{1} \leqq \varrho$

$$
\|F x\|_{1} \leqq\|\alpha\|_{1}+b\|x\|_{1} \leqq a+b \varrho
$$

and consequently $\mu_{F}(\varrho) \leqq v_{f}(\varrho)$. On the other hand, given $\varrho>0$ define $\varphi_{\varrho}: \Omega \times \mathbf{R} \rightarrow \mathbf{R}$ by

$$
\varphi_{\varrho}(s, u):=\max \left\{0,|f(s, u)|-\mu_{F}(\varrho)|u| \varrho^{-1}\right\},
$$

fix a function $x \in L_{1}(\Omega)$, and let $\Omega^{+}$denote the set of all points $s \in \Omega$ for which $\varphi_{\varrho}(s, x(s))$ is positive. Now choose $n \in \mathbf{N}$ and $\theta \in[0,1)$ such that

$$
\int_{\Omega^{+}}|x(s)| d s=(n+\theta) \varrho,
$$

and divide $\Omega^{+}$into subsets $\Omega_{1}^{+}, \ldots, \Omega_{n+1}^{+}$such that

$$
\int_{\Omega_{i}^{+}}|x(s)| d s \leqq \varrho \quad(i=1, \ldots, n+1) .
$$

From the definition of the function $\mu$ it follows then that

and therefore

$$
\int_{\Omega_{i}^{+}}|f(s, x(s))| d s \leqq \mu_{F}(\varrho) \quad(i=1, \ldots, n+1)
$$

$$
\begin{gathered}
\int_{\Omega}\left|\varphi_{\varrho}(s, x(s))\right| d s \leqq(n+1) \mu_{F}(\varrho)-\mu_{F}(\varrho) \varrho^{-1} \int_{\Omega^{+}}|x(s)| d s \\
=(n+1) \mu_{F}(\varrho)-\mu_{F}(\varrho) \varrho^{-1}(n+\theta) \varrho \leqq \mu_{F}(\varrho)
\end{gathered}
$$

such that the function $\alpha_{\varrho}(s):=\sup \left\{\varphi_{\varrho}(s, u): u \in \mathbf{R}\right\}$ lies in $L_{1}(\Omega)$,

$$
|f(s, u)| \leqq \alpha_{\varrho}(s)+b_{\varrho}|u|,
$$

and $a_{\varrho}:=\left\|\alpha_{\varrho}\right\|_{1} \leqq \mu_{F}(\varrho), b_{\varrho}:=\mu_{F}(\varrho) \varrho^{-1}$. Altogether we have shown that for each $\varrho>0$ there exists a pair $\left(a_{\varrho}, b_{\varrho}\right) \in T(f)$ satisfying $a_{\varrho}+b_{\varrho} \varrho \leqq \mu_{F}(\varrho)+\mu_{F}(\varrho) \varrho^{-1} \varrho=$ $2 \mu_{F}(\varrho)$. From this the inequality $v_{f}(\varrho) \leqq 2 \mu_{F}(\varrho)$ follows immediately.

Theorem 1 shows that the problem of obtaining upper estimates for the operator $F$ reduces to studying the set $T(f)$. If a pair $\left(a_{0}, b_{0}\right)$ belongs to this set, then so do all pairs $(a, b)$ with $a \geqq a_{0}$ and $b \geqq b_{0}$, and this point set in $\mathbf{R}^{2}$ is bounded from below by some function $\theta_{f}$, namely

$$
\theta_{f}(\lambda):=\inf \{a:(a, \lambda) \in T(f)\} .
$$

The following lemma which we cite without proof recalls the well known correspondence between the functions $v=v_{f}$ and $\theta=\theta_{f}$ : 
Lemma 1. The functions $v$ and $\theta$ satisfy the relations

$$
\begin{aligned}
& v(\varrho)=\inf \{\theta(\lambda)+\lambda \varrho: 0<\lambda<\infty\}, \\
& \theta(\lambda)=\sup \{v(\varrho)-\lambda \varrho: 0<\varrho<\infty\} .
\end{aligned}
$$

Moreover, $v$ is nonnegative, nondecreasing, and concave, whereas $\theta$ is nonnegative, nonincreasing, and convex.

Let us consider some examples. If, for instance,

$$
v(\varrho):=M \varrho^{\alpha} \quad(M>0, \quad 0<\alpha<1),
$$

then

conversely, if

$$
\theta(\lambda)=(1-\alpha) \alpha^{-\beta} M^{(1-\beta)} \lambda^{\beta} \quad\left(\alpha^{-1}+\beta^{-1}=1\right) ;
$$

$$
\theta(\lambda):=N \lambda^{\beta} \quad(N>0, \quad-\infty<\beta<0),
$$

then

$$
v(\varrho)=(1+\beta) \beta^{-\alpha} N^{(1-\alpha)} \varrho^{\alpha} \quad\left(\alpha^{-1}+\beta^{-1}=1\right) .
$$

Another example is provided by

for which one has

$$
v(\varrho):=\log (\varrho+1)
$$

$$
\theta(\lambda)=\max \{0, \lambda-1-\log \lambda\} .
$$

As a corollary of the preceding results we get the following two lemmas:

Lem ma 2. The following four statements are equivalent $\left(0<\alpha<1, \alpha^{-1}+\beta^{-1}=1\right)$ :

(a) $\|F x\|_{1}=o\left(\|x\|_{1}^{\alpha}\right) \quad\left(\|x\|_{1} \rightarrow 0\right.$ or $\left.\|x\|_{1} \rightarrow \infty\right)$,

(b) $\mu_{F}(\varrho)=o\left(\varrho^{\alpha}\right) \quad(\varrho \rightarrow 0$ resp. $\varrho \rightarrow \infty)$,

(c) $v_{f}(\varrho)=o\left(\varrho^{\alpha}\right) \quad(\varrho \rightarrow 0$ resp. $\varrho \rightarrow \infty)$,

(d) $\theta_{f}(\lambda)=o\left(\lambda^{\beta}\right) \quad(\lambda \rightarrow \infty$ resp. $\lambda \rightarrow 0)$.

Proof. Let us first prove the equivalence of (a) and (b). If (a) holds for $\|x\|_{1} \rightarrow 0$ then for each $\varepsilon>0$ there exists $\delta>0$ such that $\|x\|_{1} \leqq \delta$ implies $\|F x\|_{1} \leqq \varepsilon\|x\|_{1}^{\alpha}$. Hence, given $\varrho \leqq \delta$, one has $\|F x\|_{1} \leqq \varepsilon \varrho^{\alpha}$ for any $x \in L_{1}(\Omega)$ with $\|x\|_{1} \leqq \varrho$, which means that (b) holds for $\varrho \rightarrow 0$. Conversely, if (b) holds for $\varrho \rightarrow 0$ (i.e. $\varrho \leqq \delta$ implies $\mu_{F}(\varrho) \leqq \varepsilon \varrho^{\alpha}$ for $\varepsilon>0$ ) then, by setting $\|x\|_{1}=: \varrho$, one has

$$
\|F x\|_{1} \leqq \mu_{F}(\varrho) \leqq \varepsilon \varrho^{\alpha}=\varepsilon\|x\|_{1}^{\alpha}
$$

for $\|x\|_{1} \leqq \delta$ which means that (a) holds for $\|x\|_{1} \rightarrow 0$.

The analogous proof for $\|x\|_{1} \rightarrow \infty$ and $\varrho \rightarrow \infty$, respectively, is somewhat more complicated: If (b) holds for $\varrho \rightarrow \infty$, then for each $\varepsilon>0$ there exists $\omega>0$ such that

$$
\sup \left\{\|F x\|_{1}:\|x\|_{1} \leqq \varrho\right\} \leqq \varepsilon \varrho^{\alpha} \quad(\varrho \geqq \omega)
$$


therefore, for any $x \in L_{1}(\Omega)$ with $\|x\|_{1}=: \varrho \geqq \omega$ we have

$$
\|F x\|_{1} \leqq \varepsilon \varrho^{\alpha}=\varepsilon\|x\|_{1}^{\alpha} .
$$

Conversely, suppose that (a) holds for $\|x\|_{1} \rightarrow \infty$, i.e. given $\varepsilon>0$ there exists $\tau>0$ such that $\|x\|_{1} \geqq \tau$ implies $\|F x\|_{1} \leqq \varepsilon\|x\|_{1}^{\alpha}$. We must find $\omega>0$ such that (6) holds; to this end, we consider two cases: On the one hand, if $\mu_{F}(\tau) \leqq \varepsilon \tau^{\alpha}$ then $\varrho \geqq \omega:=\tau$ implies

$$
\|F x\|_{1} \leqq \begin{cases}\varepsilon \tau^{\alpha} \leqq \varepsilon Q^{\alpha} & \|x\|_{1} \leqq \tau, \\ \varepsilon\|x\|_{1}^{\alpha} \leqq \varepsilon Q^{\alpha} & \tau \leqq\|x\|_{1} \leqq \varrho .\end{cases}
$$

On the other hand, if $\mu_{F}(\tau)>\varepsilon \tau^{\alpha}$, then choose $\omega:=(\sigma / \varepsilon)^{1 / \alpha}$ where

$$
\sigma:=\sup \left\{\|F x\|_{1}:\|x\|_{1} \leqq \tau,\|F x\|_{1}>\varepsilon \tau^{x}\right\}<\infty
$$

and observe that $\omega>\tau$. In this case $\varrho \geqq \omega$ implies

$$
\|F x\|_{1} \leqq \begin{cases}\sigma=\varepsilon \omega^{\alpha} \leqq \varepsilon Q^{\alpha} & \|x\|_{1} \leqq \tau, \\ \varepsilon\|x\|_{1}^{\alpha} \leqq \varepsilon \omega^{\alpha} \leqq \varepsilon Q^{\alpha} & \tau \leqq\|x\|_{1} \leqq \omega, \\ \varepsilon\|x\|_{1}^{\alpha} \leqq \varepsilon Q^{\alpha} & \omega \leqq\|x\|_{1} \leqq \varrho,\end{cases}
$$

which proves (6) and hence the equivalence of (a) and (b).

The equivalence of (b) and (c) follows from Theorem 1.

Assume now, for example, that $(d)$ holds without loss of generality for $\lambda \rightarrow \infty$, (the other three implications between (c) and (d) are dealt with similarly), i.e.

$$
\lim _{\lambda \rightarrow \infty} \theta_{f}(\lambda) \lambda^{-\beta}=0 .
$$

Let $\varepsilon>0$ be given; we will find $\delta>0$ such that $0<\varrho<\delta$ implies

$$
v_{f}(\varrho) \varrho^{-\alpha}=\inf _{\lambda}\left\{\theta_{f}(\lambda)+\lambda \varrho\right\} \varrho^{-\alpha}<\varepsilon,
$$

i.e. for each $\varrho<\delta$ we will find $\lambda(\varrho)>0$ such that

$$
\theta_{f}(\lambda(\varrho)) \varrho^{-\alpha}+\lambda(\varrho) \varrho^{1-\alpha} \leqq \varepsilon .
$$

Because of (7), we can find $\omega>0$ such that

$$
\theta_{f}(\lambda) \lambda^{-\beta}<\varepsilon / 2
$$

for $\lambda \geqq \omega$. Furthermore, the choice $\delta:=\min \left\{\omega^{\beta / \alpha},(\varepsilon / 2)^{1 /(1-\beta)}\right\}$ yields $\varrho^{\alpha / \beta}>\delta^{\alpha / \beta} \geqq \omega$ for $\varrho<\delta$ (note that $\beta$ is negative!). Hence, if we set $\lambda(\varrho):=\varrho^{\alpha / \beta}$, then $\lambda(\varrho) \geqq \omega$, i.e.

$$
\begin{gathered}
\theta_{f}(\lambda(\varrho)) \lambda(\varrho)^{-\beta} \varrho^{-\alpha}+\lambda(\varrho)^{1-\beta} \varrho^{1-\alpha} \\
<\frac{\varepsilon}{2} \varrho^{-\alpha}+\varrho^{(1-\beta) \alpha / \beta+1-\alpha}=\frac{\varepsilon}{2} \varrho^{-\alpha}+\varrho^{1-\beta-\alpha} \\
=\left[\frac{\varepsilon}{2}+\varrho^{1-\beta}\right] \varrho^{-\alpha}<\left[\frac{\varepsilon}{2}+\delta^{1-\beta}\right] \varrho^{-\alpha} \leqq \varepsilon \varrho^{-\alpha}=\varepsilon \lambda(\varrho)^{-\beta} .
\end{gathered}
$$

Multiplying these inequalities by $\lambda(\varrho)^{\beta}$ yields the desired relation (8). 
Lemma 3. The following four statements are equivalent $\left(0<\alpha<1, \alpha^{-1}+\beta^{-1}=1\right)$ :

(a) $\|F x\|_{1}=O\left(\|x\|_{1}^{\alpha}\right) \quad\left(\|x\|_{1} \rightarrow 0\right.$ or $\left.\|x\|_{1} \rightarrow \infty\right)$,

(b) $\mu_{F}(\varrho)=O\left(\varrho^{\alpha}\right) \quad(\varrho \rightarrow 0$ resp. $\varrho \rightarrow \infty)$,

(c) $v_{f}(\varrho)=O\left(\varrho^{\alpha}\right) \quad(\varrho \rightarrow 0$ resp. $\varrho \rightarrow \infty)$,

(d) $\theta_{f}(\lambda)=O\left(\lambda^{\beta}\right) \quad(\lambda \rightarrow \infty$ resp. $\lambda \rightarrow 0)$.

Proof. Since the proof is in the same spirit as that of Lemma 2 (the equivalence between (c) and (d) is even more straightforward here) we shall ommit it.

Instead, we wish to make an important observation:

Remark 1. If we consider the first condition of Lemma 3 for $\alpha=1$, i.e. (a') $\|F x\|_{1}=O\left(\|x\|_{1}\right)$

then it is not hard to see that this condition is equivalent to the assertion that

$\left(\mathrm{a}^{\prime \prime}\right) \quad|f(s, u)| \leqq k(s)|u|$

for some $k \in L_{\infty}(\Omega)$. However, in general the condition

$\left(\mathrm{a}^{\prime \prime \prime}\right) \quad|f(s, u)| \leqq k(s)|u|^{\alpha}$

(for $\alpha<1$ ) is only sufficient for (a) but not necessary, as can be seen by the following counterexample $([1])$ :

Let $\Omega=(0,1), 0<\alpha<1, \alpha^{-1}+\beta^{-1}=1$, and

$$
f(s, u):= \begin{cases}s^{\beta}|u|\left(1-\log s^{\beta}|u|\right) & |u| \leqq s^{-\beta} \\ 1 & |u|>s^{-\beta}\end{cases}
$$

Then $F x(s)=f(s, x(s))$ maps the space $L_{1}(\Omega)$ into itself (just take $\alpha(s)=\exp \left(-b s^{-\beta}\right)$ in (1)); on the other hand, the function $f(s, u)|u|^{-\alpha}$ behaves like $\eta(s)=s^{\alpha \beta}$ (for $\left.|u|>s^{-\beta}\right)$ which is not essentially bounded on $(0,1)$ since $\alpha \beta<0$.

This counterexample shows that growth conditions on the function $f$ and the operator $F$ may be not equivalent, at least when the growth of $f$ is nonlinear (i.e. $\alpha \neq 1$ in $\left(\mathrm{a}^{\prime \prime \prime}\right)$ ).

\section{The general case}

So far we have restricted ourselves to the case $p=q=1$. In general, the superposition operator $F$ maps $L_{p}(\Omega)$ into $L_{q}(\Omega)(1 \leqq p, q<\infty)$ if and only if there is a pair $(a, b)$ of positive numbers such that

$$
|f(s, u)| \leqq \alpha(s)+b \mid u^{p / q} \quad(s \in \Omega, u \in \mathbf{R})
$$

for some function $\alpha \in L_{q}(\Omega)$ with $\|\alpha\|_{q} \leqq a$. This general case, however, can be 
reduced to the special one by setting

$$
\tilde{f}(s, u):=\left|f\left(s,|u|^{1 / p} \operatorname{sgn} u\right)\right|^{q} .
$$

Since then

$$
|\tilde{f}(s, u)| \leqq \tilde{\alpha}(s)+\tilde{b}|u|
$$

with $\tilde{\alpha}(s)=2^{q} \alpha(s)$ and $\tilde{b}=2^{q} b, \tilde{F} x(s)=\tilde{f}(s, x(s))$ maps the space $L_{1}(\Omega)$ into itself. This observation allows one to formulate and prove analogous results for the general case, where, of course, the scalar functions introduced above must be modified appropriately. Thus, if we set now

$$
v_{f}(\varrho):=\inf \left\{a+b \varrho^{p / q}:(a, b) \in T(f ; p, q)\right\}
$$

(where $T(f ; p, q)$ is the set of pairs $(a, b)$ used in (9)), then Lemma 1 holds, with $\lambda \varrho$ replaced by $\lambda \varrho^{p / q}$. Moreover, Theorem 1 reads now as follows:

Theorem 2. The function

$$
\mu_{F}(\varrho)=\sup \left\{\|F x\|_{q}:\|x\|_{p} \leqq \varrho\right\}
$$

and $v_{f}$ (given by (11)) are equivalent in the sense that

$$
\mu_{F}(\varrho) \leqq v_{f}(\varrho) \leqq 2 \mu_{F}(\varrho) .
$$

Proof. The assertion can be proved either by adapting the proof of Theorem 1 (with $\varphi_{\varrho}$ given by

$$
\left.\varphi_{\varrho}(s, u):=\max \left\{0,|f(s, u)|-\mu_{F}(\varrho)|u|^{p / q} \varrho^{-p / q}\right\}\right)
$$

or by applying the transformation (10).

Finally, we state the following generalization of Lemma 2 as a separate theorem (Lemma 3 generalizes in exactly the same way):

Theorem 3. Suppose that the operator $F$ maps $L_{p}(\Omega)$ into $L_{q}(\Omega)$. Then the following four statements are equivalent $\left(0<\alpha<p / q, \beta^{-1}+\alpha^{-1} p / q=1\right)$ :

(a) $\|F x\|_{q}=o\left(\|x\|_{p}^{\alpha}\right) \quad\left(\|x\|_{p} \rightarrow 0\right.$ or $\left.\|x\|_{p} \rightarrow \infty\right)$

(b) $\mu_{F}(\varrho)=o\left(\varrho^{\alpha}\right) \quad(\varrho \rightarrow 0$ resp. $\varrho \rightarrow \infty)$,

(c) $v_{f}(\varrho)=o\left(\varrho^{\alpha}\right) \quad(\varrho \rightarrow 0$ resp. $\varrho \rightarrow \infty)$,

(d) $\theta_{f}(\lambda)=o\left(\lambda^{\beta}\right) \quad(\lambda \rightarrow \infty$ resp. $\lambda \rightarrow 0)$.

Proof. Using the notation (10), one easily shows that

$$
\mu_{\widetilde{F}}(\varrho)=\mu_{F}\left(\varrho^{1 / p}\right)^{q}
$$

and hence (b) is equivalent to $\mu_{\tilde{F}}(\varrho)=o\left(\varrho^{\alpha q / p}\right)$. According to Lemma 2 , this in turn is equivalent to $v_{\tilde{f}}(\varrho)=o\left(\varrho^{\alpha q ! p}\right)$, and hence to (c), since

$$
v_{\tilde{f}}(\varrho) \leqq 2^{q} v_{f}\left(\varrho^{1 / p}\right)^{q} \leqq 2^{2 q} v_{\tilde{f}}(\varrho) .
$$


The equivalence between (a) and (b) is established similarly by reducing the problem to the operator $\tilde{F}$.

Finally, the equivalence between (c) and (d) follows from the definition (11) by applying the same reasoning as in Lemma 2 .

Let us point out once more that (by Remark 1) the condition

$$
|f(s, u)| \leqq k(s) \mid u^{\alpha} \quad\left(k \in L_{\infty}(\Omega), \quad 0<\alpha<p / q\right)
$$

is only sufficient for

but not necessary.

$$
\|F x\|_{q} \leqq k\|x\|_{p}^{\alpha} \quad(k>0)
$$

Finally, we wish to note that analogous problems can be studied not only in $L_{p}$ spaces but, more generally, in Orlicz spaces. For example, if $L_{M}(\Omega)$ and $L_{N}(\Omega)$ are two Orlicz spaces (where $N$ satisfies a $\Delta_{2}$ condition), then the superposition operator $F$ (generated by $f$ ) maps the unit ball of $L_{M}(\Omega)$ into $L_{N}(\Omega)$ if and only if the superposition operator $G$ (generated by the function $g(s, u)=$ $\left.N\left(f\left[s, M^{-1}(u)\right]\right)\right)$ maps the space $L_{1}(\Omega)$ into itself ([4], [5]). Therefore from some suitable transformation like (10) one can derive estimates for $F$ between $L_{M}(\Omega)$ and $L_{N}(\Omega)$. These problems will be dealt with in a forthcoming paper.

\section{Differentiability conditions}

There is a rather easy way to derive differentiability conditions for the superposition operator $F$ from Theorem 3. To this end, observe that, given a nonlinear operator $F$ between two Banach spaces $X$ and $Y$, the existence of the derivative $F^{\prime}\left(x_{0}\right)$ at $x_{0} \in X$ can be expressed in the form

$$
\left\|F\left(x_{0}+x\right)-F\left(x_{0}\right)-F^{\prime}\left(x_{0}\right) x\right\|_{Y}=o\left(\|x\|_{X}\right) \quad\left(\|x\|_{X} \rightarrow 0\right)
$$

while the existence of the asymptotic derivative $F^{\prime}(\infty)$ means that

$$
\left\|F(x)-F^{\prime}(\infty) x\right\|_{Y}=o\left(\|x\|_{X}\right) \quad\left(\|x\|_{X} \rightarrow \infty\right) .
$$

In [9] it is shown that if $F: L_{p}(\Omega) \rightarrow L_{q}(\Omega)(p>q)$ is differentiable at zero with derivative $G=F^{\prime}(0)$ (and $F(0)=0$, without loss of generality), then necessarily the "limit in measure"

$$
g(s):=\mu-\lim _{v \rightarrow 0} f(s, v) v^{-1}
$$

exists and belongs to $L_{p q /(p-q)}(\Omega)$, and $G$ has the form $G x(s)=g(s) x(s)$. On the other hand, several sufficient conditions were given in [3] and [5] as well as in [9] which are, however, not necessary. Similarly, a necessary condition for the 
existence of the asymptotic derivative $F^{\prime}(\infty)$ is that the limit

$$
g(s):=\mu-\lim _{v \rightarrow \infty} f(s, v) v^{-1}
$$

exist and be an $L_{p q /(p-q)}(\Omega)$ function.

We can state here a differentiability condition which is both necessary and sufficient:

Theorem 4. Let $f$ be a Carathéodory function such that $f(s, 0) \equiv 0$ and $F$ maps $L_{p}(\Omega)$ into $L_{q}(\Omega)(p>q)$. Then $F$ is differentiable at 0 with derivative $G=F^{\prime}(0)$ [or asymptotically linear with asymptotic derivative $G=F^{\prime}(\infty)$ ] if and only if the following two conditions are fulfilled:

(i) the limit (14) (resp. the limit (15)) exists and belongs to $L_{p q /(p-q)}(\Omega)$,

(ii) for each $\lambda>0$ there exists a function $a_{\lambda} \in L_{q}(\Omega)$ such that $\left\|a_{\lambda}\right\|_{q}=o\left(\lambda^{-q /(p-q)}\right)$ for $\lambda \rightarrow \infty$ (resp. $\lambda \rightarrow 0)$ and

$$
|f(s, u)-g(s) u| \leqq a_{\lambda}(s)+\lambda|u|^{p / q} .
$$

Moreover, the connection between $g$ and $G$ is given by $g=G x_{1}\left(x_{1}(s) \equiv 1\right)$ and $G x(s)=g(s) x(s)$.

Proof. We will prove the theorem only for the derivative at zero, the other part follows exactly the same scheme.

A. Sufficiency: If conditions (i) and (ii) are satisfied, then for each $\varepsilon>0$ there exists $\omega>0$ such that $\left\|a_{\lambda}\right\|_{q} \leqq \varepsilon^{p /(p-q)} \lambda^{-q /(p-q)}$ for $\lambda \geqq \omega$. Consequently, if we choose $\lambda:=\varepsilon\|x\|_{p}^{-(p-q) / q}$ and $\|x\|_{p}$ is sufficiently small, then $\lambda \geqq \omega$ and hence $\left\|a_{\lambda}\right\|_{q} \leqq \varepsilon\|x\|_{p}$; altogether, we have

$$
\begin{aligned}
\|F x-G x\|_{q} & \leqq \\
& =2 \varepsilon a_{\lambda}\left\|_{q}+\lambda\right\| x \|_{p}
\end{aligned}
$$

which means that $\|F x-G x\|_{q}=o\left(\|x\|_{p}\right)$ or, equivalently, $\mu_{F-G}(\varrho)=o(\varrho)(\varrho \rightarrow 0)$.

B. Necessity: If $F$ is differentiable at $0 \in L_{p}(\Omega)$, then according to the above mentioned result, condition (i) is fulfilled and $G x(s)=g(s) x(s)$. Hence, given $\varepsilon>0$, there exists $\delta>0$ such that $\|x\|_{p} \leqq \delta$ implies $\|F x-G x\|_{q} \leqq \varepsilon\|x\|_{p}$. From Theorem 3 $\mathrm{it}$ follows that the function

$$
a_{\lambda}(s):=\sup _{u} \max \left\{0,|f(s, u)-g(s) u|-\lambda|u|^{p / q}\right\}
$$

belongs to $L_{q}(\Omega)$ and satisfies $\left\|a_{\lambda}\right\|_{q} \leqq \varepsilon \lambda^{-q /(p-q)}$ which means that (ii) holds.

Theorem 4 admits a generalization to higher derivatives. There are several possibilities to define higher order derivatives of a nonlinear operator $F: X \rightarrow Y$. The "Taylor-series version" states that $F$ has derivatives up to order $m$ at $x_{0} \in X$ if

$$
\left\|F\left(x_{0}+x\right)-F\left(x_{0}\right)-G_{1} x-\frac{1}{2} G_{2} x-\ldots-\frac{1}{m !} G_{m} x\right\|_{Y}=o\left(\|x\|_{X}^{m}\right),
$$


where the operators $G_{k}$ are homogeneous of order $k$, i.e. $G_{k}(\lambda x) \equiv \lambda^{k} G x$. Using this notion one obtains the following criterion for higher differentiability which is proved in the same way as Theorem 4:

Theorem 5. Let $f$ be a Carathéodory function such that $f(s, 0) \equiv 0$ and $F$ maps $L_{p}(\Omega)$ into $L_{q}(\Omega)(p>m q)$. Then $F$ is m-times differentiable at 0 with derivatives $G_{k}=F^{(k)}(0)(k=1, \ldots, m)$ if and only if the following two conditions are fulfilled:

(i) the limits

$$
g_{k}(s)=k ! \mu-\lim _{v \rightarrow 0} v^{-k}\left[f(s, v)-g_{1}(s) v-\ldots-\frac{g_{k-1}(s)}{(k-1) !} v^{k-1}\right]
$$

$(k=1, \ldots, m)$ exist and belong to $L_{p q /(p-k q)}(\Omega)$,

(ii) for each $\lambda>0$ there exists a function $a_{\lambda} \in L_{q}(\Omega)$ such that $\left\|a_{\lambda}\right\|_{q}=$ $o(\lambda-m q /(p-m q))$ for $\lambda \rightarrow \infty$ and

$$
\left|f(s, u)-g_{1}(s) u-\ldots-g_{m}(s) u^{m}\right| \leqq a_{\lambda}(s)+\lambda|u|^{p / q} .
$$

Moreover, the connection between $g_{k}$ and $G_{k}$ is given by $g_{k}=G_{k} x_{1}\left(x_{1}(s) \equiv 1\right)$ and $G_{k} x(s)=g_{k}(s)[x(s)]^{k}$.

\section{Applications}

Let us briefly indicate how to apply the results of the preceding sections to the solvability of the Hammerstein integral equation

$$
\begin{aligned}
x(s)-A x(s) & =x(s)-\int_{\Omega} k(s, t) f(t, x(t)) d t \\
& =y(s) .
\end{aligned}
$$

As usual, we assume that $y \in L_{p}(\Omega), F$ maps $L_{p}(\Omega)$ into $L_{q}(\Omega)$ with $f(s, 0) \equiv 0$, and the linear integral operator

$$
K x(s)=\int_{\Omega} k(s, t) x(t) d t
$$

maps $L_{q}(\Omega)$ continuously into $L_{p}(\Omega)$. The solvability of equation (16) relies usually on the application of either Schauder's fixed point principle (and its generalizations) or other methods related to derivatives of $A$. In all these cases $A$ is assumed to be compact, condensing, etc.

In order to apply fixed point principles one must find invariant closed convex bounded sets. If we assume for simplicity that these invariant sets are balls with center at the origin and radius $\varrho>0$, then the condition

$$
\|K\| \mu_{F}(\varrho) \leqq \varrho
$$

is sufficient for this ball to be invariant under $A$. Instead of using the function 
$\mu_{F}$ it is more convenient to use the function $v_{f}$ defined in (11) which is much easier to calculate. In this way, the problem of finding an invariant ball is reduced to the problem of solving the scalar inequality

$$
\|K\| v_{f}(\varrho) \leqq \varrho .
$$

Nevertheless, for most applications it is still more practical to use differentiability properties of $A$ rather than fixed point properties. All existence results of this type ([3], [6], [7]) use the existence of the asymptotic derivative and the fact that 1 is not an eigenvalue of $A^{\prime}(\infty)$. If $y=0$ in (16) (i.e. we consider the homogeneous equation), then the problem $x=A x$ always has the trivial solution $x=0$. To prove the existence of another nontrivial solution one assumes usually that the derivative $A^{\prime}(0)$ at zero exists. In this case, if the sum of the multiplicities of the real eigenvalues $>1$ of $A^{\prime}(0)$ and $A^{\prime}(\infty)$, respectively, have different parity (i.e. are not both even or odd, see [3]), then the homogeneous equation $x=A x$ has a nontrivial solution. Obviously, when making use of this type of theorem, one must prove the differentiability of $A$ at 0 or $\infty$. Theorem 4 allows us to give a precise formulation of differentiability and, consequently, to enlarge the field of applications.

Integral operators of Hammerstein type play an important role in various fields of nonlinear oscillations. For example, some of the problems on forced vibrations in automatic control systems which can be described by equations of the form

$$
L(p) x=M(p) f(t, x)
$$

(where $L(p)$ and $M(p)$ are linear differential operators with constant coefficients of order $l>1$ and $m<l$, respectively) lead to equation (16). If the function $f$ is periodic with respect to time $t$ with the period $T>0$, the $T$-periodic solutions to equation (17) coincide with the solutions to equation (16), where $\Omega=[0, T]$, and the kernel $k$ has the form $k(t, s)=G(t-s)$, with $G$ being the so-called impulsefrequency characteristic of the linear term with transform function

$$
W(p)=\frac{M(p)}{L(p)} .
$$

Similarly, such integral equations are related to certain problems on self-excited vibrations; in these problems the kernel of the integral operator (16) and the nonlinearity often depend on various parameters (where the periods of the self-excited vibrations are not known a priori).

In this way, our results allow us to obtain new existence theorems for periodic solutions to equation (17).

The author expresses his deep gratitude to Professor P. P. Zabreĭko for his constant interest and very valuable advice during the preparation of this paper. 


\title{
References
}

[1] Appell, J.: Implicit functions, nonlinear integral equations, and the measure of noncompactness of the superposition operator. - J. Math. Anal. Appl. 83, 1981, 251-263.

[2] Krasnosel'skir̆, M. A.: The continuity of the operator $f u(x)=f[x, u(x)]$. - Doklady Akad. Nauk SSSR (N. S.) 77, 1951, 185-188 (Russian).

[3] Krasnosel'skil̆, M. A.: Topological methods in the theory of non-linear integral equations. Gosudarst. Izdat. Techn.-Teor. Lit., Moscow, 1956 (Russian). (English translation: Pergamon Press, Oxford-London-New York-Paris, 1964.)

[4] KRASnosel'skin̆, M. A. and YA. B. Rutickiř: Convex functions and Orlicz spaces. - Gosudarstv. Izdat. Fiz.-Mat. Lit., Moscow, 1958 (Russian). (English translation: P. Noordhoff Ltd., Groningen, 1961.)

[5] Krasnosel'skil̆, M. A., P. P. Zabrě̌ko, E. I. Pustyl'nik, and P. E. SobolevskiǏ: Integral operators in spaces of summable functions. - Izdat. "Nauka”, Moscow, 1966 (Russian). (English translation: Noordhoff Int. Publ., Leyden, 1976.)

[6] Martelli, M., and A. Vignoli: Eigenvectors and surjectivity for $\alpha$-Lipschitz mappings in Banach spaces. - Ann. Mat. Pura Appl. (4) 94, 1972, 1-9.

[7] Pejsachowicz, J., and A. Vignoli: On differentiability and surjectivity of $\alpha$-Lipschitz mappings. - Ann. Mat. Pura Appl. (4) 101, 1974, 49-63.

[8] Ruticklı̆, YA. B.: On a nonlinear operator acting on an Orlicz space. - Dopovidi Akad. Nauk Ukrain. RSR 3, 1952, 161-166 (Ukrainian. Russian summary).

[9] WANG Sheng-WANG: Differentiability of the Nemyckir operator. - Dokl. Akad. Nauk SSSR 150, 1963, 1198-1201 (Russian).

\author{
Freie Universität Berlin \\ 1. Mathematisches Institut \\ Hüttenweg 9 \\ D 1000 Berlin 33
}

Received 14 September 1982 